\title{
Role of contrast-enhanced FLAIR MRI in diagnosis of intracranial lesions
}

\author{
Wessam Mustafa ${ }^{1} \mathbb{D}$, Sherif Ali $^{2}$, Nadia Elgendy ${ }^{3}$, Samer Salama ${ }^{1 *} \mathbb{D}$, Lamiaa El Sorogy ${ }^{2}$ and Mohamed Mohsen ${ }^{2}$
}

\begin{abstract}
Background: MR imaging plays a significant role in detection and characterization of different brain diseases. The role of the post-contrast T1-weighted image magnetic resonance imaging (T1W MRI) sequence has been widely established in previous studies and clinical practice. In this study, we aim to share our experience as regards the added value of contrast-enhanced fluid-attenuated inversion recovery (CE-FLAIR) sequence in the diagnosis of various intracranial pathological conditions and evaluate its usefulness in comparison with post-contrast T1W images.

Results: Based on the final radiological diagnosis, the total cases were subdivided into three categories, and the majority of our cases were tumors (81.2\%), followed by multiple sclerosis (11.8\%), and the least was central nervous system infection (7.1\%). CE-FLAIR showed superior enhancement in 35 cases (50.7) and equal enhancement in 25 cases (36.3\%). However, it showed less enhancement than post-contrast T1W images in 9 cases (13\%). Excellent inter-observer agreement (97.65\%) was noted. Regarding lesion conspicuity, good delineation was found in the majority of cases (64.7\%), fair delineation in $12.9 \%$, and no delineation in $22.4 \%$. A statistically significant difference was found in signal intensity of lesion between pre- and post-contrast FLAIR sequences. Contrast to background ratio was statistically significant in CE FLAIR images in comparison to CE T1 images.

Conclusion: CE-FLAIR imaging should be used as a routine or adjunctive sequence to CE-T1WI to enhance early detection and increase the diagnostic confidence in MRI examination of different brain pathological conditions.

Keywords: Contrast-enhanced FLAIR (CE-FLAIR), Intracranial lesions, MRI sequences, Brain
\end{abstract}

\section{Background}

Intravenous contrast agents for magnetic resonance imaging (MRI) are commonly used to allow proper lesion recognition and classification of Central nervous system (CNS) lesions. Gadolinium is the most commonly used contrast agent, as it can shorten both the T1 and T2 relaxation times of tissues where the contrast is present. However, enhancement of the lesion via contrast occurs primarily by the T1-shortening consequence at clinical doses [1].

Fluid-attenuated inversion recovery (FLAIR) is a "special inversion recovery pulse sequence with a long repetition time (TR) and echo time (TE), and an inversion

\footnotetext{
*Correspondence: samer_salama@mans.edu.eg

${ }^{1}$ Department of Neurology, Mansoura University, Mansoura, Egypt Full list of author information is available at the end of the article
}

time (TI) that effectively nulls signals from the cerebrospinal fluid (CSF)" [1, 2].

The post-gadolinium enhancement that appears on T2-fluid attenuated inversion recovery (T2 FLAIR) images is due to the $\mathrm{T} 2$ prolongation consequence of multiple lesions and T1 shortening consequence of gadolinium acting together [3].

Previous studies established the value of contrastenhanced fluid-attenuated inversion recovery (FLAIR) sequence in imaging of leptomeningeal metastases, which may be attributed to three main factors. First, the FLAIR sequence does not allow contrast enhancement of vessels. Second, it reveals an observable T1 contrast with a dark CSF signal. Third, T2 FLAIR with contrast is more sensitive than T1-weighted image (T1WI) in detecting low concentrations of gadolinium [4]. 
The greatest advantage of contrast-enhanced FLAIR (CE-FLAIR) seems to be for detecting subtle cortical abnormalities such as leptomeningeal lesions, where there is no mass effect. However, it also has an advantage when compared with conventional FLAIR or T2weighted imaging for deep lesions, where the contrast between the enhancing lesion and the surrounding vasogenic edema is greater than with conventional techniques and produces very remarkable images [5].

The aim of this study is to assess the role of CEFLAIR imaging as a primary or adjunctive sequence to contrast-enhanced T1-weighted image (CE-T1WI) to increase the diagnostic confidence.

\section{Methods}

Our prospective study had been conducted on 85 patients (males and females) who were referred from the neuro-medicine and neurosurgery departments to MRI unit of the radio-diagnosis department, in the period from December 2018 till August 2020.

All patients with solitary space-occupying lesion (SOL) were pathologically confirmed by tissue biopsy and the patient with multiple SOLs was diagnosed by follow-up or biopsy of extracranial primary tumors. Imaging diagnoses of inflammatory lesions were confirmed by clinical signs, laboratory findings, and follow-up. Informed written consents were obtained from all participants in the study.

MRI imaging was performed on 1.5-T magnetic resonance scanner (Philips Achieva 2.1, Best, Netherlands) with standard head coil for the brain and with the patient in a supine position. Routine conventional MRI ( axial fast spin echo (FSE) T1-weighted images, T2 weighted image, fluid attenuation inversion recovery (FLAIR), diffusion-weighted images, and apparent diffusion coefficient) post-contrast images were obtained after administration of intravenous Gadolinium (Magnevist) in the dose of $0.1 \mathrm{mmol} / \mathrm{kg}$ body weight. One minute after the intravenous administration of contrast medium, the acquisition of coronal, sagittal, and axial routine post-contrast T1W images was immediately done, and then axial or coronal T2W FLAIR images were successively obtained with a delay of nearly 3-4 min and with the same scan techniques as the precontrast images.

Contrast-enhanced T1W imaging parameters were repetition time (TR) $581 \mathrm{~ms}$, echo time (TE) $15 \mathrm{~ms}$, field of view (FOV) $230 \mathrm{~mm}$, slice thickness $5 \mathrm{~mm}$, slice interval $1 \mathrm{~mm}$, and flip angle 69 .

Contrast-enhanced FLAIR imaging parameters were TR $11,000 \mathrm{~ms}$, TE $110 \mathrm{~ms}$, TI $2800 \mathrm{~ms}$, FOV $230 \mathrm{~mm}$, slice thickness $5 \mathrm{~mm}$, and slice interval $1 \mathrm{~mm}$.

Images interpretation was performed by the assessment of each case independently by two radiologists
(AA, RA). Images were initially interpreted as usual brain MRI to detect any gross pathology: multiple sclerosis (Fig. 1), infection (Fig. 2), tumor (Fig. 3), etc. using the conventional sequences. Then post-contrast T1WI (PC-T1W) and post-contrast FLAIR MRI sequences were compared with each other (Figs. 1, 2, and 3). Images were evaluated both qualitatively and quantitatively.

Qualitative evaluation (Fig. 4) by visual assessment includes the following items: Presence or absence of abnormal contrast enhancement, determining the location and pattern of enhancement, comparing the results of information detected by CE FLAIR with other sequences, evaluation of enhancement rate in postcontrast T1W and post-contrast FLAIR MRI sequences, and classifying it in post-contrast FLAIR images as superior, equivocal, or inferior relative to post-contrast T1W images, delineation of enhanced lesion margin in post-contrast FLAIR images from surrounding edema, or normal-appearing brain tissue, referred to as lesion conspicuity was evaluated as either: no delineation, fair delineation or good delineation and correlating the imaging findings with the clinical data.

Quantitative evaluation (Fig. 5) was done by: calculating contrast enhancement index (CEI) by placing region of interest (ROI) tool at site of lesion to measure signal intensity (SI) in pre- and post-contrast FLAIR images. Contrast enhancement index (CEI): $I^{\wedge}=\mathrm{I}-\mathrm{I} 0$. Where $I^{\wedge}$ is $\mathrm{CEI} I$ is the lesion signal intensity (SI) on post-contrast FLAIR images and IO is the lesion SI on pre-contrast FLAIR images. Calculating lesion to background contrast ratio: The signal intensity of the lesion as well as background signal intensity in normal-appearing brain tissue was measured by using the region-of-interest (ROI) tool and applied to pre- and post-contrast T1W as well as pre- and post-contrast FLAIR sequences. The lesion to background contrast ratio was defined as the difference between the lesion and background signal intensities divided by the background signal intensity (Fig. 6).

\section{Statistical analysis and data interpretation}

Data were fed to the computer and analyzed using Statistical Package for the Social Sciences (IBM SPSS) Corp. Released 2013. IBM SPSS Statistics for Windows, Version 22.0. Armonk, NY: IBM Corp. Qualitative data were analyzed in the form of numbers and percentage. While, Quantitative data were assessed through median (minimum and maximum) as regard non-parametric data and mean, standard deviation to those of the parametric data, this was done only after completion of testing normality via Kolmogrov-Smirnov test. Assessment of the test significance of results was done at the (0.05) level.

Qualitative data, Monte Carlo test as correction for Chi-Square test when more than $25 \%$ of cells have count less than 5 in tables $(>2 \times 2)$. Quantitative data between 


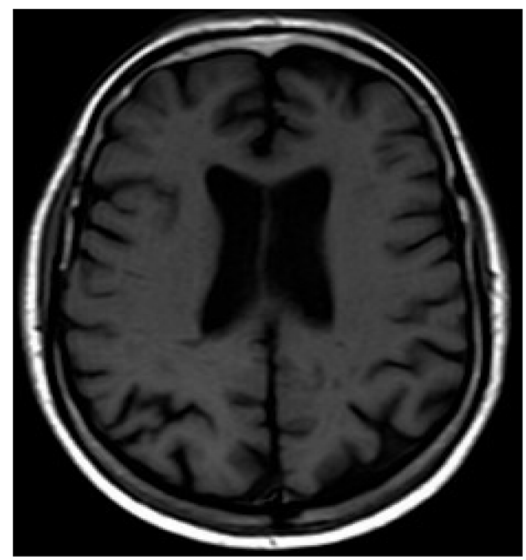

(A)

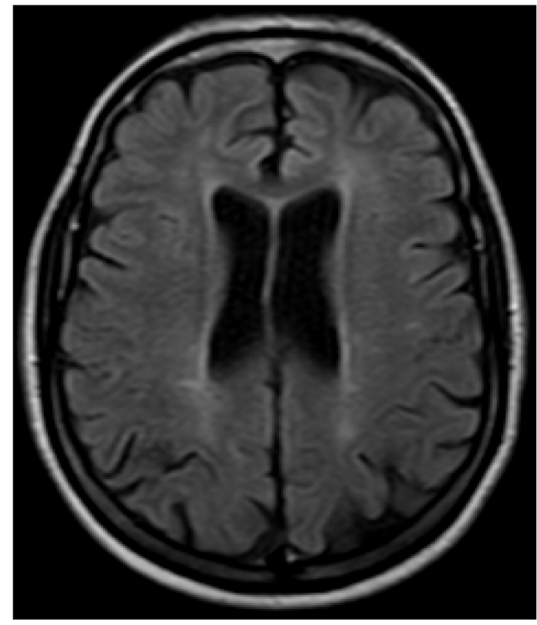

(C)

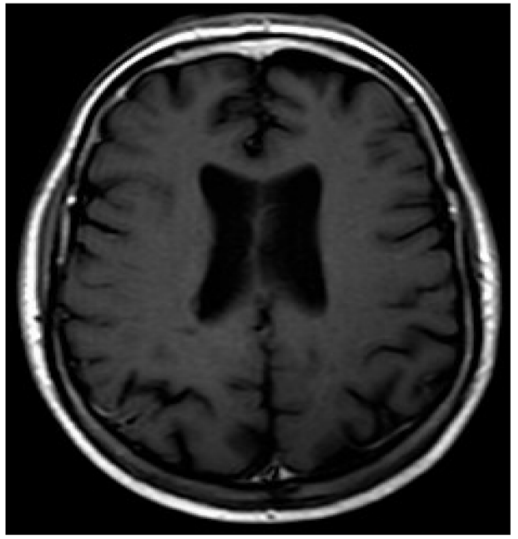

(B)

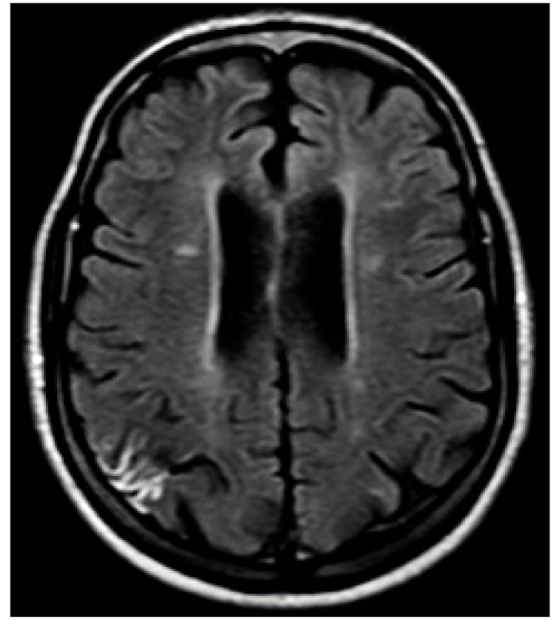

(D)

Fig. 1 Female patient aged 36 years, known case of multiple sclerosis on follow up, presented by clinical signs of activity. A, B Axial pre and postcontrast T1WI respectively showed no definite lesions. C Axial pre-contrast FLAIR showed few foci of high signal intensity at periventricular white matter. D Axial post-contrast FLAIR showed nodular enhancement of MS plaques (arrows) and gyriform enhancement at left parietal lobe was better demonstrated than in post-contrast T1W image

groups: Non Parametric tests: Mann-Whitney $U$ test was used to compare two independent groups.

The Spearman rank-order correlation is used to determine the strength and direction of a linear relationship between two non-normally distributed continuous variables and / or ordinal variables.

Inter-class correlation (ICC) was used to detect agreement between continuous variables with a correlation coefficient more than 0.7 was considered excellent agreement. The ICCs were classified using a system suggested by McGraw and Wong as follows: less than 0.75 $Z$ poor agreement, 0.75 to less than $0.90 Z$ moderate agreement, and 0.90 or greater $Z$ high agreement.
Kappa agreement was calculated by cross tabulation for categorical variables with Kappa (0.01-0.20: slight agreement, 0.21-0.40: fair agreement, 0.41-0.60: moderate agreement, 0.61-0.80: substantial agreement, and 0.81-0.99: 0 perfect agreement).

\section{Results}

This study was carried out on 85 patients, 54 females (63.5\%) and 31 males (36.5\%). The age of patients ranged from 12 to 70 years and the mean age was $41.35 \pm 15.62$. According to the final radiological diagnosis, cases were subdivided into three categories, the majority of our 


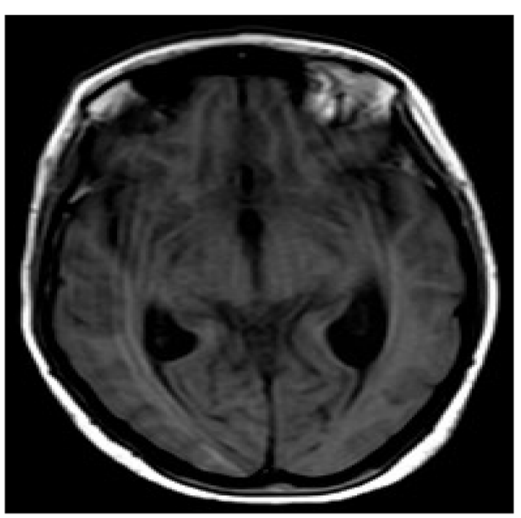

(A)

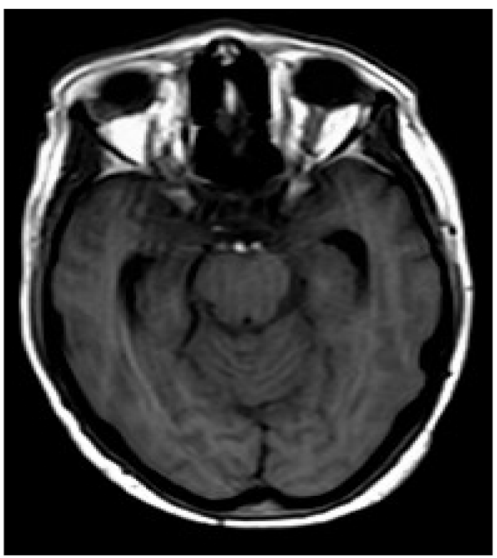

(B)

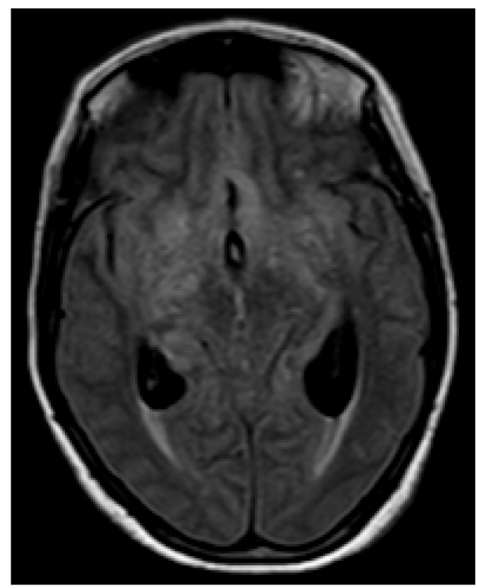

(E)

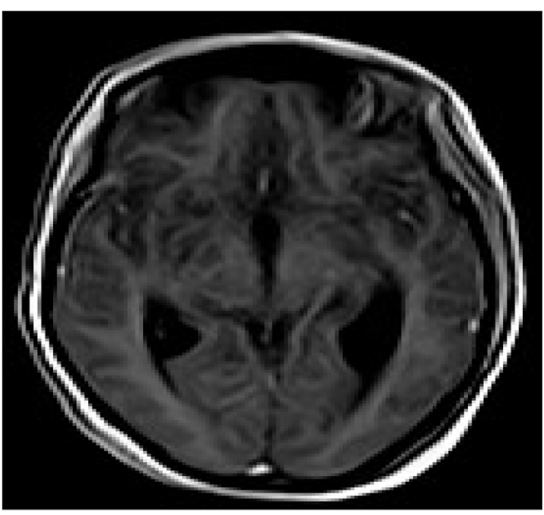

(C)

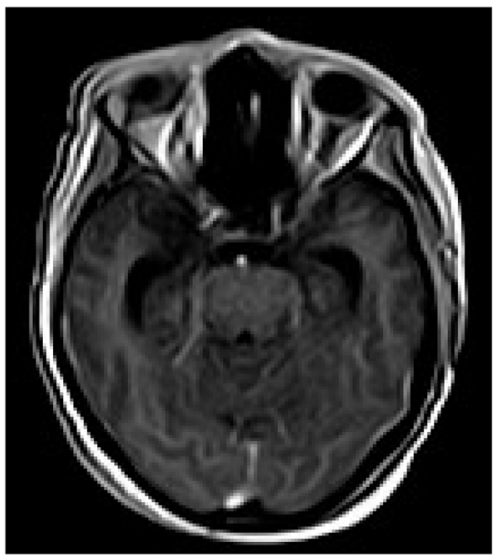

(D)

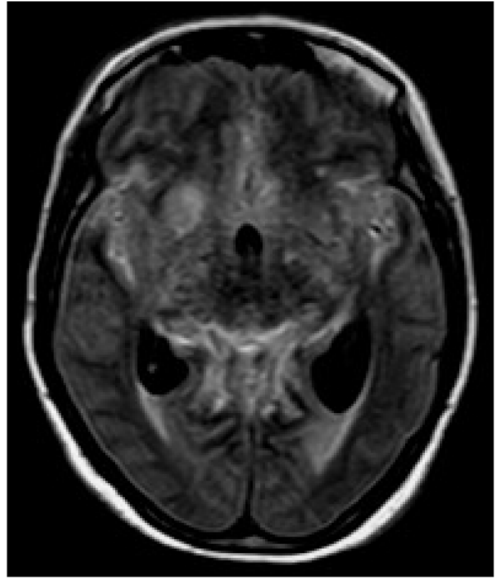

(F)

Fig. 2 (See legend on next page.) 
(See figure on previous page.)

Fig. 2 Female patient aged 35 years diagnosed as viral meningoencephalitis. A, B Axial pre-contrast T1WI showed areas of parenchymal hypointensity at the medial aspect of both temporal lobes as well as inferior frontal lobes and both insular cortices. C, D Axial post-contrast T1WI showed mild leptomeningeal enhancement at per mesencephalic cisterns. E Axial pre contrast FLAIR image showed areas of parenchymal hyperintensity at the medial aspect of both temporal lobes as well as inferior frontal lobes and both insular cortices. F Axial post-contrast FLAIR image showed marked leptomeningeal enhancement at per mesencephalic cisterns and around both Sylvian fissures, which is more extensive and more clearly delineated than in post-contrast T1WI

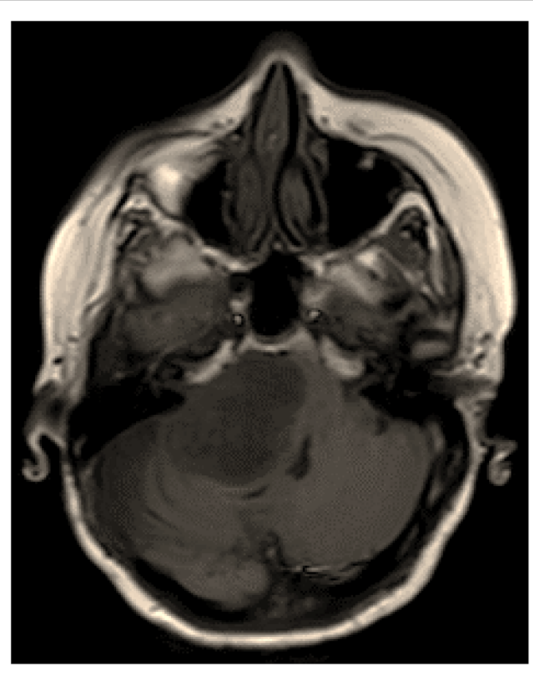

(A)

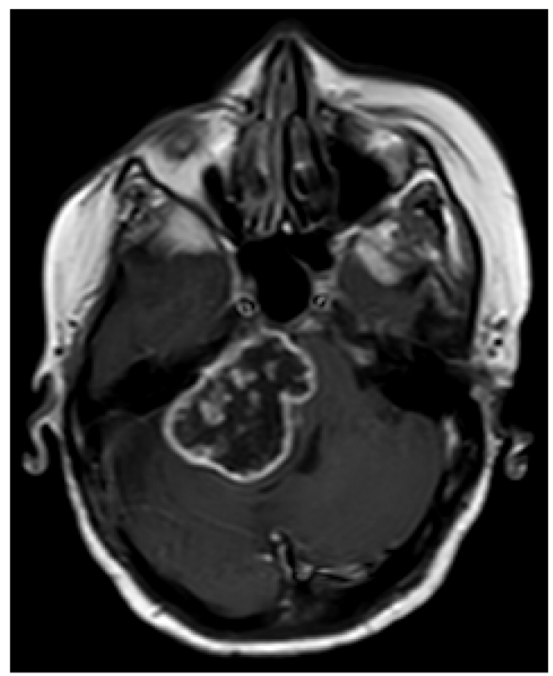

(C)

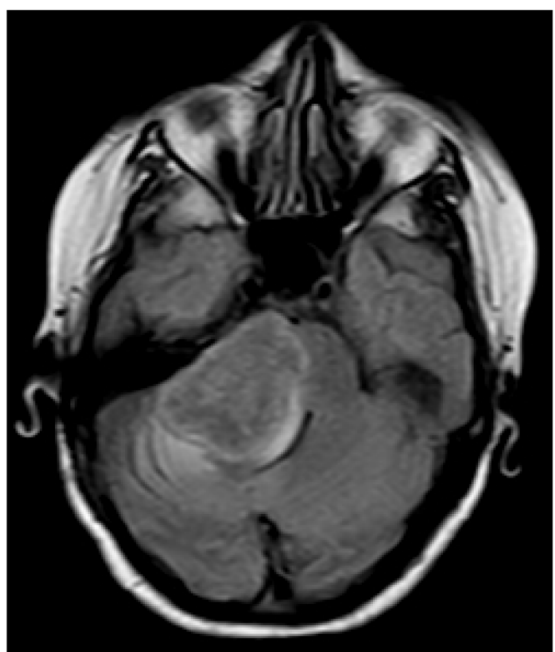

(B)

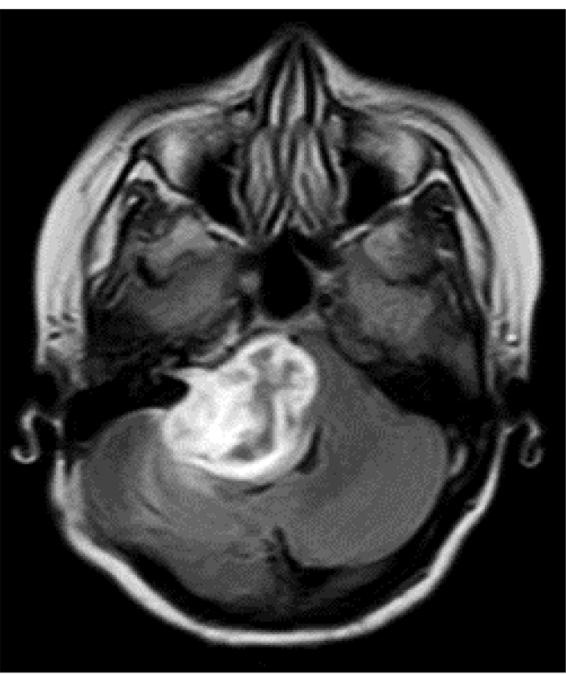

(D)

Fig. 3 Male patient aged 26 years with histopathologically proven right-sided vestibular schwannoma. A, B Axial pre-contrast T1WI and FLAIR respectively showed well defined extra-axial space-occupying lesion centered at the right cerebellopontine angle (CPA). It displayed low signal intensity on T1WI and intermediate signal intensity on FLAIR image. C Axial post-contrast T1WI showed peripheral non-homogenous enhancement with predominant internal non-enhancing cystic component. D Axial post-contrast FLAIR showed more extensive heterogeneous enhancement of the mass signifying a more solid component of the tumor. In addition, intra-canalicular extension into the internal auditory canal was more clearly demonstrated (trumpeting of the porus acousticus) 


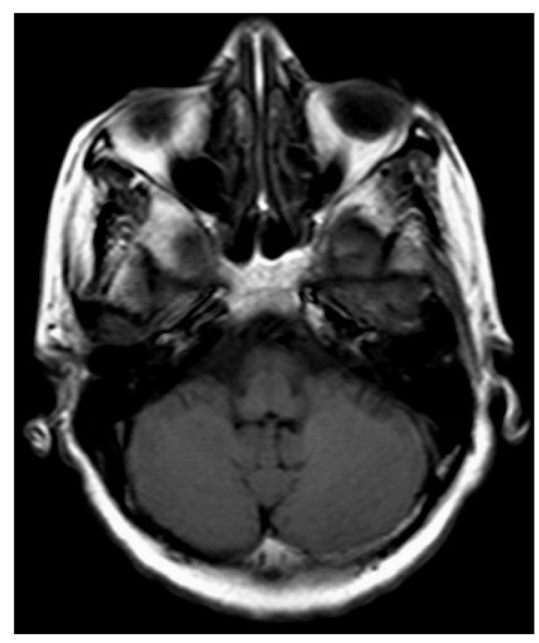

(A)

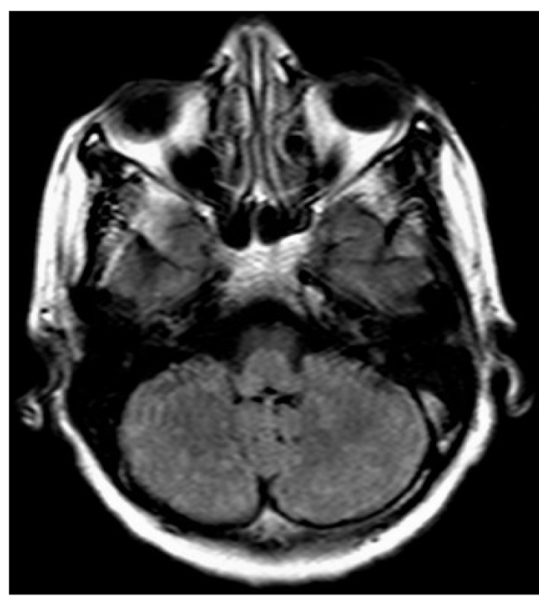

(C)

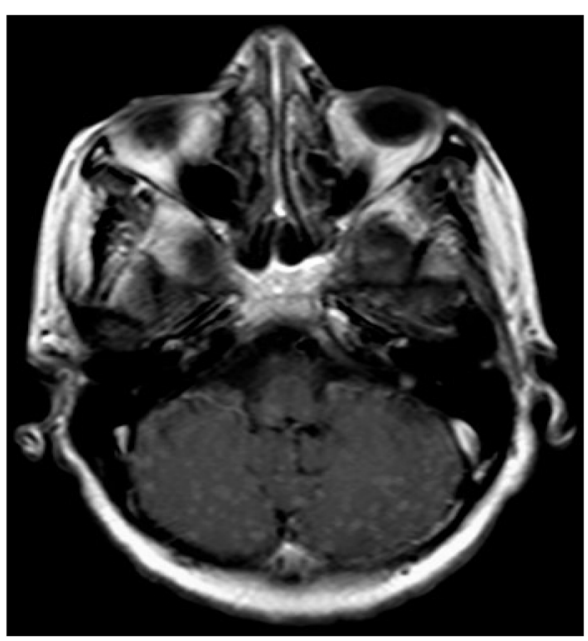

(B)

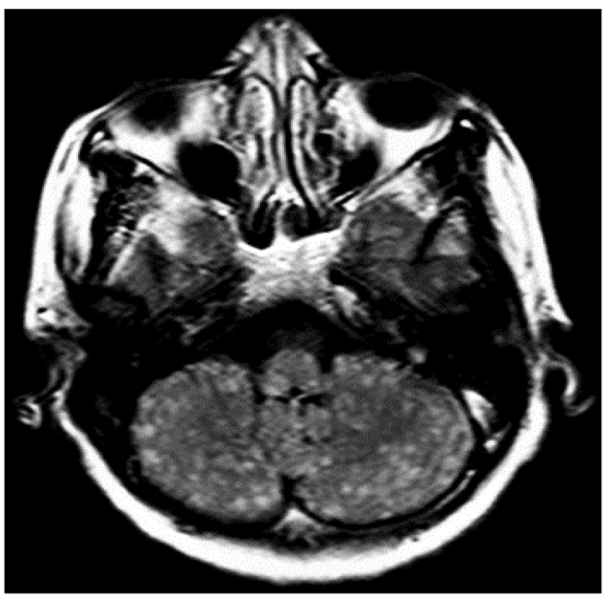

(D)

Fig. 4 Male patient aged 57 years known as bronchogenic carcinoma with brain metastases. A, B Axial pre and post contrast T1WI showed multiple tiny enhancing lesions scattered at both cerebellar hemispheres and medulla. On axial pre-contrast FLAIR (C), the lesions were few and displayed faint high signal intensity. D Axial post-contrast FLAIR images showed an increased number of enhancing lesions that appeared more hyperintense and demonstrated better lesion to background contrast than in post-contrast T1W1 (B)

cases were tumors $(81.2 \%)$, followed by multiple sclerosis (11.8\%) and the least was CNS infection (7.1\%).

According to the location of the enhanced lesion, cases were classified as having intra-axial location (70.6\%) representing the majority of studied cases, extra-axial location(23.5\%), or both intra and extra-axial location (5.9\%) (Table 1).

Qualitative comparison between post-contrast T1 and post-contrast FLAIR images revealed that: post contrast FLAIR shows superior enhancement in 35 cases (50.7), equal enhancement in 25 cases (36.3\%). However, it showed less enhancement than post-contrast T1W images in nine cases $(13 \%)$.
There was excellent agreement between the first and second observers as regard qualitative comparison of post-contrast FLAIR and post-contrast T1W sequences with Kappa agreement (0.957) and percentage of agreement (97.65\%) (Table 2).

Lesion conspicuity representing delineation of enhancing lesion from the surrounding high signal edema as well as normal nearby brain tissue in post-contrast FLAIR images was described in three groups as follows: good delineation was found in the majority of cases $(64.7 \%)$, fair delineation in $12.9 \%$, and no delineation in $22.4 \%$. 


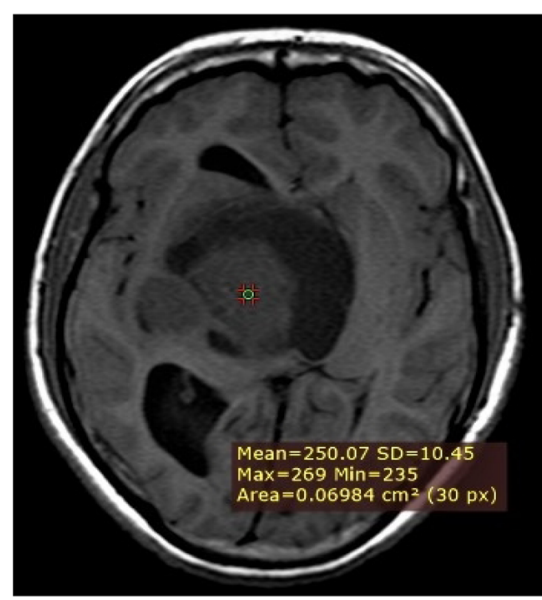

(A)

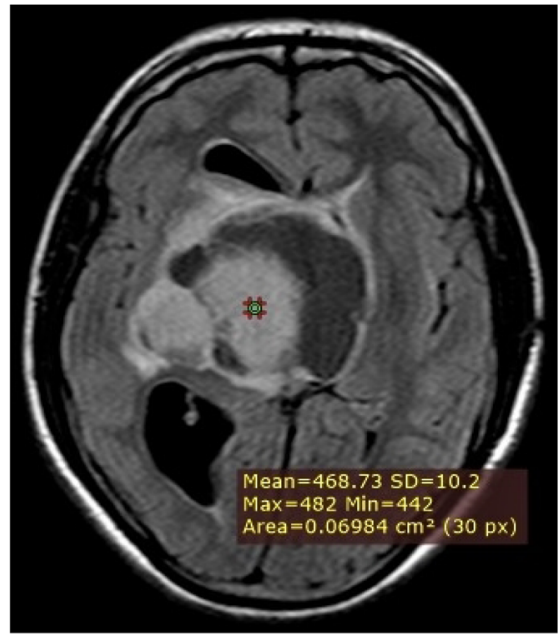

(C)

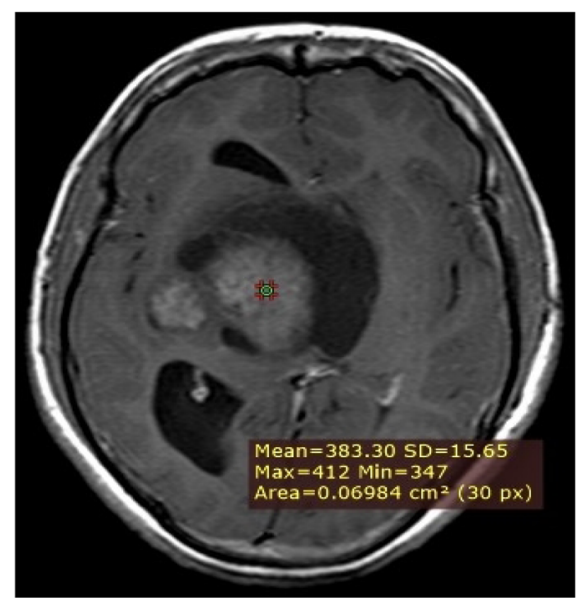

(B)

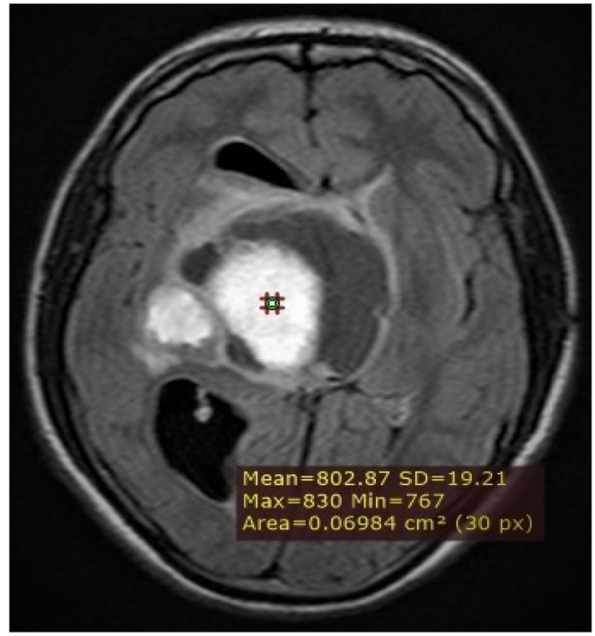

(D)

Fig. 5 Male patient aged 15 years with histopathologically proven craniopharyngioma presented on pre and post-contrast T1WI MRI (A and $\mathbf{B}$ respectively) as large well-defined suprasellar cystic lesion with enhancing solid component was noted. The lesion compresses the optic chiasm and third ventricle. On axial pre- and post-contrast FLAIR ( $\mathbf{C}$ and $\mathbf{D}$ ), qualitative and quantitative enhancement of the solid component was much more extensive in post-contrast FLAIR than in post-contrast T1WI with superior contrast to background ratio in post-contrast FLAIR sequence

There was a statistically significant difference in signal intensity of lesion between pre- and post-contrast FLAIR sequences with higher median (750) ranging from 369 to 3508 found in post-contrast FLAIR images. The median difference between signal intensity in both sequences described as contrast enhancement index (CEI) was 262 ranging from 40 up to 1708 .

Regarding the contrast ratio between lesion to the background, Table 3 showed that the contrast of lesion against related background was statistically higher in $\mathrm{CE}$ FLAIR images compared to non-contrast FLAIR images, with a higher median (0.97) ranging from 0.20 to 3.0 found in CE FLAIR images. Also, the contrast of lesion against related background was statistically higher in post-contrast $\mathrm{T} 1$ images compared to non-contrast $\mathrm{T} 1$ images, with a higher median (0.33) ranging from -0.05 to 1.2 found in post-contrast $\mathrm{T} 1$ images.

Quantitative comparison as regard contrast to background ratio was statistically significant in CE FLAIR images in comparison to CE T1 images, with a higher median (0.97) ranging from 0.20 to 3.0 in CE FLAIR images.

There was good agreement between two observers as regard signal intensity readings in pre- and post-contrast 


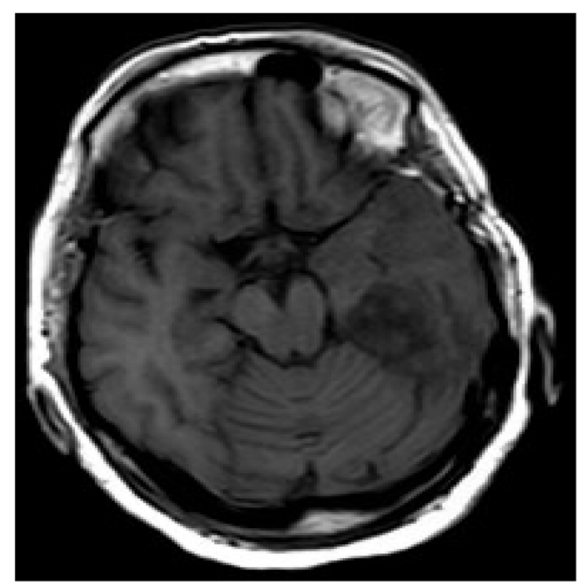

(A)

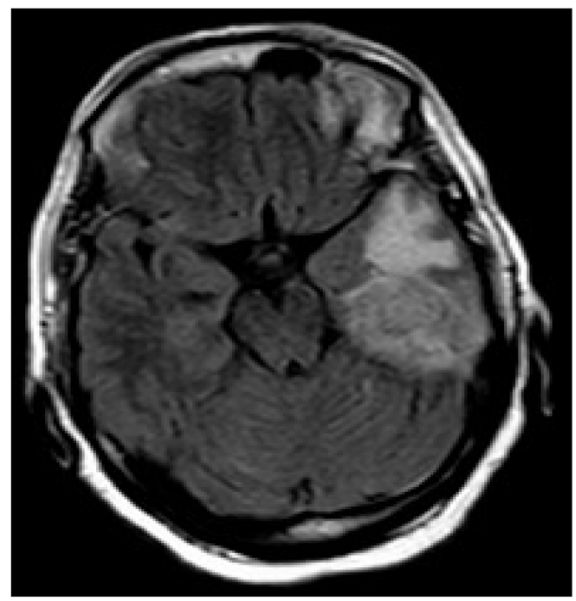

(C)

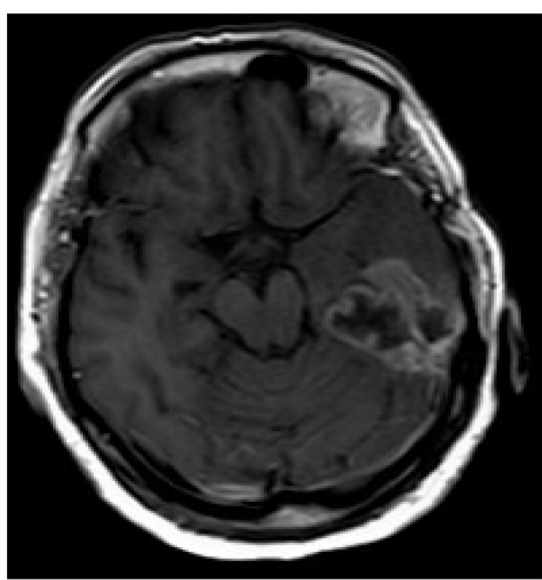

(B)

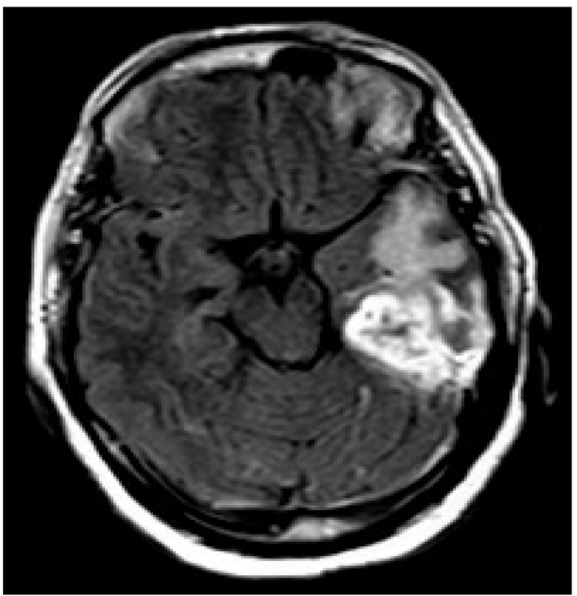

(D)

Fig. 6 Female patient aged 43 years histopathologically proven high-grade glioma. A, B axial pre- and post-contrast T1WI respectively showed intra-axial space-occupying lesion at left temporal lobe with irregular marginal enhancement. C, D Axial pre and post contrast FLAIR respectively showed thicker and more extensive enhancement of the lesion as well as better lesion to background signal intensity and overall contrast than in post-contrast T1WI. Also, good conspicuity of enhanced lesion margin from surrounding edema can be clearly demonstrated

FLAIR images. Also, contrast enhancement index as well as contrast to background ratio on post-contrast FLAIR and post-contrast T1W images with correlation coefficient more than 0.7 was considered excellent agreement (Table 4).

\section{Discussion}

Intravenous contrast agents are commonly used to evaluate patients with suspected intracranial lesions. Gadolinium is the most commonly used intravenous contrast agent for imaging the brain. It helps in improved lesion detection and better characterization [6].
No one can deny the fact of the importance of T1 post-contrast significance in detection of spaceoccupying lesions in the brain. Unfortunately, small groups in our practice are aware of the role of T2FLAIR post-contrast imaging in specific situations. Although these effects are known by some expertises, it was not used commonly in the practice. So the result is that we have small studies assessing the role of postcontrast T2 FLAIR images in different brain lesions [7].

Post-contrast T2 FLAIR imaging sequences have been investigated in a variety of intracranial pathologies with the goal of increasing diagnostic sensitivity. The diagnostic value of post-contrast-T2 FLAIR has been variably 
Table 1 variability of tumors included in the study

\begin{tabular}{lll}
\hline & $N=44$ & $\%$ \\
\hline Primary tumors & 22 & \\
Glioma & 7 & 50.0 \\
Astrocytoma & 1 & 15.9 \\
PNET & 11 & 2.3 \\
Meningioma & 3 & 25.0 \\
Schwanomma & $n=25$ & 6.8 \\
Metastatic tumors & 13 & \\
Breast & 4 & 52.0 \\
Lung & 4 & 16.0 \\
Pelvi-abdominal & 4 & 16.0 \\
Hemopioetic & & 16.0 \\
\hline PNET primitive neuro-ectodermat tumors
\end{tabular}

PNET primitive neuro-ectodermal tumors

reported in both veterinary, and human studies, with its use in meningeal-based disease commonly reported as beneficial [8].

A total of 85 cases with different intracranial pathologies were enrolled; they were further classified on the basis of the clinical diagnosis, pathological evaluation, and final imaging diagnosis into 3 groups as follows: tumors (either primary or metastatic) were found in 69 cases $(81.2 \%)$ representing most of our studied cases, with 44 cases $(63.8 \%)$ primary tumors and 25 cases (36.2\%) metastatic tumors; Multiple sclerosis was observed in 10 cases (11.8\%); and infection (including meningitis, meningoencephalitis, and brain abscess) was detected in 6 cases $(7.1 \%)$.

In our study, we provided additional subtraction imaging between post and corresponding pre-contrast FLAIR sequences; this was beneficial and provided improvement in detection of very small enhancing lesions, or lesions of initially high signal intensity in nonenhanced FLAIR images as well as in cases where there is an improper delineation of enhancing lesion margin from the hyperintense perilesional edema. In addition, we found that the use of subtraction imaging helped avoid false-positive cases, decreased reading time, and increased the accuracy of detection of contrast enhancement in a clinical practice, and this was equivalent to the results of the study done by Zivadinov and his colleagues [9].

Results of qualitative comparison between the two sequences showed that CE-T2 FLAIR provided superior enhancement in 49 cases (57.6\%), less enhancement was detected in 10 cases (11.8\%), yet equivocal enhancement in both sequences was found in 26 cases (30.6\%). These results are in agreement with those obtained by Athar and colleagues who demonstrated better enhancement in 19 cases $(57.6 \%)$ in post-contrast T2 FLAIR weighted images, less enhancement in 6 cases $(18.2 \%)$. However, in the remaining 8 cases $(24.2 \%)$, both sequences do not reveal any abnormality so considered as equal [10].

Lesion conspicuity, delineation of enhancing lesion margin from the surrounding normal brain tissue or perilesional edema as well as the ability to clearly identify enhancement and lesion outline in post-contrast FLAIR sequence were evaluated in all cases and described as either: good delineation, reported in 55 cases (64.7\%), fair delineation, reported in 11 cases (12.9\%) and no delineation, reported in 19 cases (22.4\%). Our results for good delineation are relatively higher than the results of the study done by Athar and colleagues that reported a clear outline of a lesion in post-contrast FLAIR sequence in 13 cases (39.4\%) out of 33 cases. This could be explained by different sample sizes.

In order to avoid any bias in qualitative analysis of post-contrast FLAIR images, the signal intensity of all lesions was measured in both pre- and post-contrast FLAIR images and the difference was calculated and expressed as contrast enhancement index (CEI). Our results demonstrated significantly higher signal intensity on CE-FLAIR images compared to pre-contrast ones $(P$ $<0.001)$, which confirm the presence of enhancement after contrast injection.

In this study, lesion-to-background contrast percentage was assessed objectively to prevent making subjective data via visual assessment of contrast that might be affected by windows and levels, amplification, and monitor illumination.

Our study demonstrated a significant higher contrast ratio in CE-FLAIR compared to both non-enhanced FLAIR and CE-T1W sequences $(P<0.001)$ which allowed better delineation of lesions by CE-FLAIR. The higher contrast ratio detected by CE-FLAIR can be explained by greater inherent soft tissue contrast resolution in FLAIR compared to T1W sequence. In addition, unlike CE-T1WI, CE-FLAIR images normal vasculature and healthy meninges do not enhance. Therefore, CE-FLAIR images are considered as being

Table 2 Inter-observer agreement of qualitative comparison with PCT1

\begin{tabular}{lllllll}
\hline Qualitative comparison with PCT1W & First observer & Second observer & $\mathbf{K}$ & $\mathbf{p}$ & $\mathbf{9 5 \%} \mathbf{C l}$ & $\mathbf{\%}$ of agreement \\
\hline Equal & 26 & 25 & 0.957 & $<0.001^{*}$ & $0.89-1.0$ & $97.65 \%$ \\
Inferior & 10 & 9 & & & & \\
Superior & 49 & 51 & & & & \\
\hline
\end{tabular}

K Kappa agreement, $\mathrm{Cl}$ confidence interval, PCT1W post-contrast T1-weighted image 
Table 3 Lesion to background contrast ratio

\begin{tabular}{llll}
\hline Background contrast ratio & Mean \pm SD & Median (range) & Test of significance \\
\hline NC FLAIR & $0.342 \pm 0.25$ & $0.3(-0.31,1.0)$ & $z=8.0$ \\
CE FLAIR & $1.08 \pm 0.60$ & $0.97(0.20-3.0)$ & $p<0.001^{*}$ \\
NC T1 & $-0.13 \pm 0.15$ & $-0.13(-0.60,0.43)$ & $z=7.57$ \\
CE T1 & $0.40 \pm 0.34$ & $0.33(-0.05,1.2)$ & $p<0.001^{*}$ \\
\hline
\end{tabular}

CE FLAIR contrast-enhanced FLAIR MRI, NC FLAIR non-contrast-enhanced FLAIR MRI, CE T1 contrast-enhanced T1-weighted image, NC T1 non-contrast-enhanced T1-weighted image. Z: Wilcoxon signed-rank test

most effective to assess sulcal or meningeal infection, inflammation, and metastases near the CSF side.

In our study, good agreement was found between two observers as regard signal intensity readings in pre- and post-contrast FLAIR images, contrast enhancement index as well as contrast to background ratio on postcontrast FLAIR and post-contrast T1W images with correlation coefficient more than 0.7 was considered excellent agreement.

In line with our study, Kim and colleagues stated that contrast-enhanced fast FLAIR images had higher tumorto-background contrast ratio compared to contrastenhanced T1W images [3].

Furthermore, ZHOU and colleagues observed that the CER and contrast-to-noise ratio (CNR) on CE T1WI was significantly higher but grey matter/white matter contrast was lower $(P=0.02)$ than those on $C E$ FLAIR images [11].

While another study by Tomura and colleagues in which the intensity ratios (intensity of tumor divided by intensity of peritumoral region) in contrast-enhanced spin echo T1W and contrast-enhanced multi-shot echoplanar imaging FLAIR (Ms-EPI-FLAIR), "comprising combined sequences of FLAIR and Ms-EPI" were compared, showed that the intensity ratio in Ms-EPI-FLAIR did not differ from that in spin echo T1WI.These results were in contrary to what we had observed [12]. This discrepancy could be attributed to different sample sizes, different imaging parameters, and different MRI machines with different specifications.

Table 4 Interobserver agreement as regard signal intensity, CEl, and contrast to background ratio

\begin{tabular}{ll}
\hline & Interclass correlation \\
\hline Signal intensity pre FLAIR & 0.78 \\
Signal intensity CE FLAIR & 0.88 \\
CEI & 0.79 \\
Lesion to background contrast ratio: & \\
CE FLAIR & 0.77 \\
CE T1 & 0.87 \\
\hline
\end{tabular}

FLAIR fluid-attenuated inversion recovery, CE FLAIR contrast-enhanced FLAIR MRl, CE T1 contrast-enhanced T1-weighted image, CEl contrast enhancement index
A study conducted by Azad and colleagues, where quantitative assessment included computation of net meningeal enhancement, using single pixel signal intensity software and comparing the results between CEFLAIR, Magnetization Transfer Spin Echo, and FatSaturation T1-Weighted Sequences, observed that a significant difference was found between the net meningeal enhancement on the contrast-enhanced FLAIR sequence compared to the magnetization transfer spin echo and the T1-weighted fat saturation sequences $(p<0.001)$ [13]. These results seem to be consistent with the data obtained by our study.

In concordance with the previous study done by Rastogi and Jain, we found that, in 69 cases of intracranial tumors post-contrast FLAIR showed superior enhancement in 35 cases (50.7\%), equal enhancement in 25 cases $(36.3 \%)$, and less enhancement than post-contrast T1W images in 9 cases (13\%) [7]. We had also observed that in patients with tumors, CE-T2FLAIR images were considered to be superior to CE-T1W images as it allow to reveal more widespread enhancement (rather than solid part) and denser and more nodular wall enhancement in the necrotic tumor. So after interpretation of these findings not only the differentiation of the tumor can be performed but also the site for stereotactic biopsy can be determined.

We had also observed that CE-FLAIR yielded significantly more information compared to routine CE-T1W sequence in detection of early leptomeningeal metastatic lesions and small superficial parenchymal lesions as well. There is no doubt that early identification of leptomeningeal disease affects the treatment and overall prognosis of many brain tumors. Our results were consistent with the study done by Kim and colleagues [2] who stated that in small intracerebral metastasis, lesion detection was increased when contrast-enhanced FLAIR was added to contrast-enhanced T1WI.

On the basis of our observations of 10 patients with multiple sclerosis, we found that CE-FLAIR was superior to CE-T1W sequence in 9 cases $(90 \%$ of our studied MS cases), and provided better detectability and significantly more number of active lesions, this was assessed by depicting active lesions as ultra-bright relative to precontrast FLAIR images and confirming enhancement by 
FLAIR subtraction images along with measuring signal intensity of MS plaques in pre- and post-contrast FLAIR images; furthermore, these findings were supported by clinical data. So, we suggest that CE-FLAIR would be a promising diagnostic technique and should be added in diagnosis and follow-up of multiple sclerosis and can be a valuable tool in monitoring disease activity. So, our results were consistent with the findings of the study done by Abdolmohammadi and colleagues who reported that in post-contrast FLAIR images it has been observed more acute MS plaques at supratentorial area than postcontrast T1W sequence which is a gold standard sequence. Moreover, they stated that post-contrast FLAIR sequence was better at lesion visualization than the DWI and post-contrast T1W sequences [14].

Leptomeningeal infiltration (LMI) in multiple sclerosis patients in MS was firstly discovered in 2004. After that several pathological studies have established the presence of immune cell aggregations in the meninges among some groups of MS patients. Pathologically, LMI is considered as abnormal immune cell aggregations in the meninges of patients with multiple sclerosis, especially those with progressive MS either in primary or secondary forms. The presence of LMI is considered as a bad prognostic outcome with more disability and higher EDSS scores. Recently, specific MRI sequences have the ability to confirm the presence of these follicles that correspond to the pathological findings [15].

In Eisele and colleagues' study, the pathologic control group demonstrated the sensitivity of post-contrast FLAIR images demonstrating leptomeningeal enhancement in all cases. In contrast, only 1 out of 112 examined patients with MS showed a single area of abnormal leptomeningeal contrast enhancement [16]. This was quite equivalent to our results.

In our study, it is interesting to note that appreciable abnormal leptomeningeal enhancement in post-contrast FLAIR sequence was described in all cases of multiple sclerosis and was not clearly demonstrated in postcontrast T1W sequence.

Regarding 6 cases diagnosed with infection, our results showed that CE-FLAIR demonstrated superior enhancement and additional valuable reliable information compared to conventional CE-T1W sequence in 5 cases (83.3\%). We had observed that CE-FLAIR has a great capability for better detection and delineation of even subtle meningeal enhancement compared to teh $\mathrm{CE}$ T1W sequence. Furthermore, in cases of pyogenic abscess ( 2 cases), CE-FLAIR demonstrated better wall enhancement, with greater mural thickness and more clear and sharp delineation from the surrounding. These results were consistent with the studies done by Ahmad and Rastogi and Jain [7, 17] whose supported the fact that CE-FLAIR sequence has an insignificant component of vascular enhancement compared to meningeal enhancement which makes meningeal inflammation easily detected and aids in early diagnosis of infectious meningitis which is important for a favorable clinical outcome.

On the light of previously discussed results of qualitative comparison between contrast-enhanced T2 FLAIR and contrast-enhanced $\mathrm{T} 1$ sequences and by reviewing the results of multiple researchers, we concluded that contrast-enhanced FLAIR is more sensitive for subtle abnormalities than either FLAIR alone or post-contrast T1-weighted imaging alone and CE-FLAIR should be used as a valuable adjunct to conventional CE-T1W sequence when an intracranial lesion is suspected in the clinical setting.

This study has some limitations as moderate overall sample size, small percentage of patients with leptomeningeal lesions, and non-representation of other brain pathological conditions associated with leptomeningeal disease as Sturge Weber syndrome and facial and optic neuritis.

\section{Conclusion}

CE-FLAIR imaging exhibits superior enhancement and lesion detection along with better soft tissue contrast resolution compared to conventional CE-T1W sequence. We believe that it provides additional valuable diagnostic information that could affect the management and progression of the disease. Hence, it should be incorporated as a routine sequence in MRI examination of different brain pathological conditions.

\section{Abbreviations}

CE-FLAIR: Contrast-enhanced FLAIR MRI; T1W: T1-weighted image; CE-T1WI: Contrast-enhanced T1-weighted image; FLAIR: Fluid attenuated inversion recovery; TR: Repetition time; TE: Echo time; TI: Inversion time: CNS: Central nervous system; FSE: Fast spin echo; FOV: Field of view; CEI: Contrast-enhancement index; ROI: Region of interest; SI: Signal intensity; IBM SPSS: Statistical Package for the Social Sciences; ICC: Inter-class correlation; MS: Multiple sclerosis; PNET: Primitive NeuroEctodermal Tumors; PCT1: Post-contrast T1; NC: Non-contrast; CSF: Cerebrospinal fluid; CNR: Contrast-to-noise ratio; DWl: Diffusionweighted imaging; LMI: Leptomeningeal infiltration; EDSS: Expanded Disability Status Scale; MRI: Magnetic resonance imaging; SOL: Spaceoccupying lesions; Cl: Confidence interval; Sl: Signal intensity

\section{Acknowledgements \\ The authors acknowledge subjects for their participation and cooperation in this study. \\ Authors' contributions \\ W.M. and S.S. contributed in collecting data and diagnosing cases and writing the manuscript. N.E. and W.M. regularly revised the sample collection, diagnosis, analyzed the results, and prepared the manuscript in its full presentation. L.E. supervise the final work and revise the study outcomes. M.M and S.A. were involved in MRI studies and diagnosing radiological images. All authors have read and approved the final manuscript.}

\section{Funding}

This research received no specific grant from any funding agency in the public, commercial, or not-for-profit sectors. 


\section{Availability of data and materials}

The datasets generated and/or analyzed during the current study are not publicly available due to current Mansoura University regulations and Egyptian legislation but are available from the corresponding author on reasonable request and after institutional approval.

\section{Declarations}

\section{Ethics approval and consent to participate}

All procedures performed in studies involving human participants were in accordance with the ethical standards of the institutional research committee and with the 1964 Helsinki declaration and its later amendments or comparable ethical standards. The study was accepted by IRB, Faculty of Medicine, Mansoura University under the code of MS.18.10.338 by the date of 26th of October 2018.

An informed written consent was taken from each participant involved in this study prior to the conduct of any study-related activities.

All data obtained from participants were confidential and were not used outside the study. The patients had the rights to withdraw from the study at any time without giving any reason.

We agree to publish in the EJNPN and the research is only applied here.

\section{Consent for publication}

Not applicable

\section{Competing interests}

The authors declare that they have no competing interests.

\section{Author details}

'Department of Neurology, Mansoura University, Mansoura, Egypt.

${ }^{2}$ Department of Diagnostic Radiology, Mansoura University, Mansoura, Egypt.

${ }^{3}$ Faculty of Medicine, Delta University for Science and Technology, Talkha, Egypt.

Received: 18 February 2021 Accepted: 21 July 2021

Published online: 03 August 2021

\section{References}

1. Lee EK, Lee EJ, Kim S. Lee YSJKjor. Importance of contrast-enhanced fluidattenuated inversion recovery magnetic resonance imaging in various intracranial pathologic conditions. Korean J Radiol. 2016;17(1):127-41. https://doi.org/10.3348/kjr.2016.17.1.127.

2. Soyama M, Azumi RJC. A new tumor delineation method for brain metastases radiotherapy by jointly referring to contrast-enhanced T1weighted and fluid-attenuated inversion recovery MRI. Cureus. 2020;12(7): e9106.

3. Kim SC, Park S-W, Ryoo I, Jung SC, Yun TJ, Choi SH, et al. Contrast-enhanced FLAIR (fluid-attenuated inversion recovery) for evaluating mild traumatic brain injury. PLoS One. 2014;9(7):e102229.

4. Park YW, Ahn SJJIMRI. Comparison of contrast-enhanced T2 FLAIR and 3D T1 black-blood fast spin-echo for detection of leptomeningeal metastases. Investig Magn Reson Imaging. 2018;22(2):86. https://doi.org/10.13104/imri.2 018.22.2.86.

5. Oyama A, Hiraoka Y, Obayashi I, Saikawa Y, Furui S, Shiraishi K, et al. Hepatic tumor classification using texture and topology analysis of non-contrastenhanced three-dimensional T1-weighted MR images with a radiomics approach. Sci Rep. 2019;9(1):8764

6. Bhargava R, Patil AM, Bakshi V, Kalekar TM, Gandage SG. Utility of contrastenhanced fluid-attenuated inversion recovery in magnetic resonance imaging of intracranial lesions. West Afr J Radiol. 2018;25(1):34.

7. Rastogi R, Jain S, Gupta Y, Joon P, Wani A, Pratap VJ. Can postcontrast-T2 FLAIR be a boon over postcontrast-T1GRE images in MR brain imaging? J Neuroinfect Dis. 2016;7:2.

8. Dickinson PJ, Jones-Woods S. Cissell DDJVR, Ultrasound. Abrogation of fluid suppression in intracranial postcontrast fluid-attenuated inversion recovery magnetic resonance imaging: A clinical and phantom study. Vet Radiol Ultrasound. 2018;59(4):432-43. https://doi.org/10.1111/vru.12605.

9. Zivadinov R, Ramasamy D, Hagemeier J, Kolb C, Bergsland N, Schweser F, et al. Evaluation of leptomeningeal contrast enhancement using pre-and postcontrast subtraction 3D-FLAIR imaging in multiple sclerosis. AJNR Am J Neuroradiol. 2018;39(4):642-7. https://doi.org/10.3174/ajnr.A5541.
10. Athar I, Khan SA, Butt MA, Maryam S, Manzoor H, Butt MF, et al. Comparison between post contrast $\mathrm{T} 1$ weighted and $\mathrm{T} 2$ weighted flair axial sequences in various intracranial pathologies. Int J Sci Eng Res. 2019;10:872-83.

11. Zhou Z-r, Shen T-z, Chen X-r, Peng W-j. Diagnostic value of contrastenhanced fluid-attenuated inversion-recovery MRI for intracranial tumors in comparison with post-contrast T1W spin-echo MRI. Chin Med J (Engl). 2006; 119(6):467-73.

12. Tomura N, Narita K, Takahashi S, Otani T, Sakuma I, Yasuda K, et al. Contrastenhanced multi-shot echo-planar FLAIR in the depiction of metastatic tumors of the brain: comparison with contrast-enhanced spin-echo T1weighted imaging. Acta Radiol. 2007:48(9):1032-7. https://doi.org/10.1080/ 02841850701499425

13. Azad R, Tayal M, Azad S, Sharma G. Srivastava RKJKjor. Qualitative and quantitative comparison of contrast-enhanced fluid-attenuated inversion recovery, magnetization transfer spin echo, and fat-saturation T1-weighted sequences in infectious meningitis. Korean J Radiol. 2017;18(6):973-82. https://doi.org/10.3348/kjr.2017.18.6.973.

14. Abdolmohammadi J, Pourasl MH, Zandkarimi M, Sharifi K, editors. Evaluation the sensitivity of FLAIR and DWI post-inject comparision with delay enhance T1w in detection of active MS lesions 2017: J Biomed Phys Eng. 2018;8(4):365-74

15. Wicken C, Nguyen J, Karna R. Bhargava PJMs, disorders r. Leptomeningeal inflammation in multiple sclerosis: Insights from animal and human studies. Mult Scler Relat Disord. 2018;26:173-82. https://doi.org/10.1016/j.msard.2018. 09.025 .

16. Eisele $\mathrm{P}$, Griebe M, Szabo K, Wolf ME, Alonso A, Engelhardt B, et al. Investigation of leptomeningeal enhancement in MS: a postcontrast FLAIR MRI study. Neurology. 2015;84(8):770-5.

17. Ahmad A, Azad S, Azad R. Differentiation of Leptomeningeal and Vascular Enhancement on Post-contrast FLAIR MRI Sequence: Role in Early Detection of Infectious Meningitis. J Clin Diagn Res. 2015;9(1). https://doi.org/10.7860/ JCDR/2015/11519.5387.

\section{Publisher's Note}

Springer Nature remains neutral with regard to jurisdictional claims in published maps and institutional affiliations.

\section{Submit your manuscript to a SpringerOpen ${ }^{\circ}$ journal and benefit from:}

- Convenient online submission

- Rigorous peer review

- Open access: articles freely available online

High visibility within the field

- Retaining the copyright to your article

Submit your next manuscript at $>$ springeropen.com 